\title{
The dual weighted residuals approach to optimal control of ordinary differential equations
}

\author{
Karin Kraft · Stig Larsson
}

Received:date/Accepted:date

\begin{abstract}
The methodology of dual weighted residuals is applied to an optimal control problem for ordinary differential equations. The differential equations are discretized by finite element methods. An a posteriori error estimate is derived and an adaptive algorithm is formulated. The algorithm is implemented in Matlab and tested on a simple model problem from vehicle dynamics.
\end{abstract}

Keywords finite element $\cdot$ a posteriori $\cdot$ error estimate $\cdot$ adaptive $\cdot$ dual weighted residual $\cdot$ boundary value problem $\cdot$ differential-algebraic $\cdot$ vehicle dynamics

Mathematics Subject Classification (2000) $65 \mathrm{~L} 60 \cdot$ 49K15

\section{Introduction}

The aim of this work is to make a first investigation of how adaptive finite element methods can be used to solve optimal control problems. Our approach is based on an adaptive algorithm with error control based on a posteriori error estimates. The work was initiated from and motivated by a need to solve optimal control problems in vehicle dynamics.

The methodology of dual weighted residuals was developed in [1] in the context of finite element methods for partial differential equations. In this paper, we adapt the methodology to optimal control problems of the form: Find states $x$ and controls $u$

Research supported in part by the Swedish Foundation for Strategic Research through GMMC, the Gothenburg Mathematical Modelling Centre

K. Kraft

Department of Mathematical Sciences, Chalmers University of Technology and University of Gothenburg, SE-412 96 Göteborg, Sweden E-mail: karin.kraft@chalmers.se

S. Larsson

Department of Mathematical Sciences, Chalmers University of Technology and University of Gothenburg, SE-412 96 Göteborg, Sweden E-mail: stig@chalmers.se 
which

$$
\begin{array}{ll}
\text { minimize } & \mathscr{J}(x, u)=l(x(0), x(T))+\int_{0}^{T} L(x, u) d t, \\
\text { subject to } & \dot{x}(t)=f(x(t), u(t)), \quad 0<t<T, \\
& I_{0} x(0)=x_{0}, \quad I_{T} x(T)=x_{T} .
\end{array}
$$

We present an adaptive finite element method with error control based on an a posteriori error estimate which is the sum of dual weighted residuals.

Optimal control problems are solved numerically using two different approaches, the direct and the indirect [2]. In the direct approach, the problem is first discretized and a finite dimensional minimization problem is solved. In the indirect approach, the necessary conditions for optimality are determined and these equations are then solved numerically. Traditionally, the necessary conditions for optimality are derived using variational calculus [3], and their solution can be obtained using various numerical methods such as finite element methods [5] or multiple shooting [2].

In the present work we use the finite element method, in which case the direct and indirect approaches coincide. We present the classical variational calculus in a weak form and derive the necessary conditions for optimality. These consist of a system of three equations: the linearized adjoint equation for the Lagrange multiplier $z$, the original state equation for $x$, and a non-linear algebraic equation for the control variable $u$. We approximate the equations by a finite element method and derive an $a$ posteriori error representation formula and an estimate of the error in the goal functional $\mathscr{J}$. The error estimate is expressed as an elementwise sum of dual weighted residuals,

$$
\left|\mathscr{J}(x, u)-\mathscr{J}\left(x_{h}, u_{h}\right)\right| \leq \sum_{n=1}^{N}\left(R_{n}^{z} \omega_{n}^{x}+R_{n}^{x} \omega_{n}^{z}+R_{n}^{u} \omega_{n}^{u}\right)+R,
$$

where $R_{n}^{z}, R_{n}^{x}, R_{n}^{u}$ are residuals from the adjoint equation, the state equation, and the algebraic equation for the control variable, respectively, and $\omega_{n}^{x}, \omega_{n}^{z}, \omega_{n}^{u}$ are weights computed from the solutions of the respective equations indicated by the superscripts, and $R$ is a remainder which may often be neglected.

Previous work, [5], [6], aims at controlling the error in an arbitrary linear functional (or a norm) of the variables and requires the solution of an additional adjoint problem of the same size as the optimality conditions. The main advantage of the dual weighted residual error estimate is that it only uses the equations introduced in the optimality conditions and no extra dual problem has to be solved. However, it can only be used for controlling the error in the goal functional $\mathscr{J}$.

We use the error estimate as the basis for an adaptive finite element method. To simplify the implementation we use Matlab and implement the adaptive finite element method for an optimal control problem with quadratic goal functional and linear state equation. The solver is tested on an optimal control problem from vehicle dynamics. A similar method was applied to the optimal control of parabolic initial value problems in [7].

We begin in Section 2 by presenting an abstract framework for the optimal control problem where we can derive the necessary conditions for optimality as well as an $a$ posteriori representation formula for the error in the goal functional $\mathscr{J}$. In Section 3 
we apply these results to the optimal control problem. In Section 4 we specialize to a quadratic/linear optimal control problem. For this problem, we derive the a posteriori error estimate from the error representation formula and we describe the implementation of an adaptive finite element method based on the a posteriori error estimate. Finally, we solve a simple model problem from vehicle dynamics in Section 5.

\section{An abstract framework}

Following [1], we formulate the optimal control problem in an abstract way. Let $W, U, V$ be normed vector spaces, let $\dot{W} \subset W$ be a subspace, let $\hat{x} \in W$ be fixed and defne the affine space

$$
\tilde{W}=\hat{x}+\dot{W}=\{w \in W: w-\hat{x} \in \dot{W}\} .
$$

The reason for using this affine space will be clear in Section 3, where we include boundary conditions in the problem formulation. Further, we introduce smooth functionals

$$
\begin{aligned}
& \mathscr{F}: W \times U \times V \rightarrow \mathbb{R}, \\
& \mathscr{J}: W \times U \rightarrow \mathbb{R} .
\end{aligned}
$$

We assume that $\mathscr{F}(x, u ; z)$ is linear in the third variable, $z$. We use the notation that the functionals depend non-linearly on the arguments before the semicolon and linearly on the arguments after the semicolon. For example, we denote the derivative of $\mathscr{F}(x, u ; z)$ acting on a test function $\varphi_{x}$ by $\mathscr{F}_{x}^{\prime}\left(x, u ; z, \varphi_{x}\right)=\mathscr{F}_{x}^{\prime}(x, u ; z) \varphi_{x}$.

We consider optimal control problems of the form: Determine $x \in \tilde{W}$ and $u \in U$ which

$$
\begin{array}{ll}
\text { minimize } & \mathscr{J}(x, u), \\
\text { subject to } & \mathscr{F}(x, u ; \varphi)=0, \quad \forall \varphi \in V .
\end{array}
$$

The main difference with [1] is the presence of the control variable $u$, and that we need several spaces in order to allow for a Petrov-Galerkin method and non-homogeneous boundary conditions.

This is a constrained optimization problem and the necessary condition for an optimum is expressed in terms of the Lagrange functional

$$
\mathscr{L}(x, u ; z)=\mathscr{J}(x, u)+\mathscr{F}(x, u ; z), \quad(x, u, z) \in W \times U \times V .
$$

Theorem 2.1 The necessary condition for an optimum $(x, u, z) \in \tilde{W} \times U \times V$ is given by

$$
\mathscr{L}^{\prime}(x, u ; z, \varphi)=0, \quad \forall \varphi \in \dot{W} \times U \times V,
$$

that is,

$$
\begin{aligned}
\mathscr{J}_{x}^{\prime}\left(x, u ; \varphi_{x}\right)+\mathscr{F}_{x}^{\prime}\left(x, u ; z, \varphi_{x}\right) & =0, & & \forall \varphi_{x} \in \dot{W}, \\
\mathscr{J}_{u}^{\prime}\left(x, u ; \varphi_{u}\right)+\mathscr{F}_{u}^{\prime}\left(x, u ; z, \varphi_{u}\right) & =0, & & \forall \varphi_{u} \in U, \\
\mathscr{F}\left(x, u ; \varphi_{z}\right) & =0, & & \forall \varphi_{z} \in V .
\end{aligned}
$$


Proof We expand $\mathscr{L}^{\prime}$ in partial derivatives, noting that $\mathscr{L}_{z}^{\prime}\left(x, u ; z, \varphi_{z}\right)=\mathscr{F}_{z}^{\prime}\left(x, u ; z, \varphi_{z}\right)=$ $\mathscr{F}\left(x, u ; \varphi_{z}\right)$.

Note that the third equation in (2.3) is the equation in the original problem (2.1) and the first equation in (2.3) is the linearized adjoint equation.

In order to formulate a Petrov-Galerkin approximation of the equations (2.3), we assume that we have subspaces $W_{h} \subset W, \dot{W}_{h} \subset \dot{W}, V_{h} \subset V, U_{h} \subset U$, and that $\hat{x} \in W_{h}$, so that

$$
\tilde{W}_{h}=\hat{x}+\dot{W}_{h} \subset \tilde{W}
$$

The approximation of the necessary condition for optimality now becomes: find $\left(x_{h}, u_{h}, z_{h}\right) \in \tilde{W}_{h} \times U_{h} \times V_{h}$ such that

$$
\mathscr{L}^{\prime}\left(x_{h}, u_{h} ; z_{h}, \varphi\right)=0, \quad \forall \varphi \in \dot{W}_{h} \times U_{h} \times V_{h}
$$

that is,

$$
\begin{aligned}
\mathscr{J}_{x}^{\prime}\left(x_{h}, u_{h} ; \varphi_{x}\right)+\mathscr{F}_{x}^{\prime}\left(x_{h}, u_{h} ; z_{h}, \varphi_{x}\right) & =0, & & \forall \varphi_{x} \in \dot{W}_{h}, \\
\mathscr{J}_{u}^{\prime}\left(x_{h}, u_{h} ; \varphi_{u}\right)+\mathscr{F}_{u}^{\prime}\left(x_{h}, u_{h} ; z_{h}, \varphi_{u}\right) & =0, & & \forall \varphi_{u} \in U_{h}, \\
\mathscr{F}\left(x_{h}, u_{h} ; \varphi_{z}\right) & =0, & & \forall \varphi_{z} \in V_{h} .
\end{aligned}
$$

The following theorem provides an a posteriori representation formula for the error in the functional $\mathscr{J}$.

Theorem 2.2 Let $(x, u, z) \in \tilde{W} \times U \times V$ and $\left(x_{h}, u_{h}, z_{h}\right) \in \tilde{W}_{h} \times U_{h} \times V_{h}$ be solutions of (2.3) and (2.5), respectively. Then

$$
\mathscr{J}(x, u)-\mathscr{J}\left(x_{h}, u_{h}\right)=\frac{1}{2} \rho_{x}+\frac{1}{2} \rho_{z}+\frac{1}{2} \rho_{u}+R,
$$

with the residuals $\rho_{x}, \rho_{z}$, and $\rho_{u}$ defined as

$$
\begin{aligned}
& \rho_{x}=\mathscr{J}_{x}^{\prime}\left(x_{h}, u_{h} ; x-\tilde{x}_{h}\right)+\mathscr{F}_{x}^{\prime}\left(x_{h}, u_{h} ; z_{h}, x-\tilde{x}_{h}\right), \\
& \rho_{u}=\mathscr{J}_{u}^{\prime}\left(x_{h}, u_{h} ; u-\tilde{u}_{h}\right)+\mathscr{F}_{u}^{\prime}\left(x_{h}, u_{h} ; z_{h}, u-\tilde{u}_{h}\right), \\
& \rho_{z}=\mathscr{F}\left(x_{h}, u_{h} ; z-\tilde{z}_{h}\right) .
\end{aligned}
$$

Here $\left(\tilde{x}_{h}, \tilde{u}_{h}, \tilde{z}_{h}\right) \in \tilde{W}_{h} \times U_{h} \times V_{h}$ is arbitrary. The remainder term $R$ is given by

$$
\begin{aligned}
R= & \frac{1}{2} \int_{0}^{1}\left(J^{\prime \prime \prime}\left(x_{h}+s e_{x}, u_{h}+s e_{u} ; e, e, e\right)\right. \\
& \left.+\mathscr{F}^{\prime \prime \prime}\left(x_{h}+s e_{x}, u_{h}+s e_{u} ; z_{h}+s e_{z}, e, e, e\right)\right) s(s-1) d s
\end{aligned}
$$

where $e=\left(e_{x}, e_{u}, e_{z}\right) \in \dot{W} \times U \times V, e_{x}=x-x_{h}, e_{u}=u-u_{h}$, and $e_{z}=z-z_{h}$.

The remainder term is cubic in the error and can therefore often be neglected. In particular, we note that $R=0$ in the important special case when $\mathscr{F}(\cdot, \cdot ; \cdot)$ is tri-linear and $\mathscr{J}(\cdot, \cdot)$ is bi-quadratic. 
Proof We introduce the notation

$$
\begin{aligned}
\overline{\mathscr{L}}^{\prime}\left(x, x_{h}, u, u_{h} ; z, z_{h}, e\right) & =\mathscr{L}(x, u ; z)-\mathscr{L}\left(x_{h}, u_{h} ; z_{h}\right) \\
& =\int_{0}^{1} \frac{d}{d s} \mathscr{L}\left(x_{h}+s e_{x}, u_{h}+s e_{u} ; z_{h}+s e_{z}\right) d s \\
& =\int_{0}^{1} \mathscr{L}^{\prime}\left(x_{h}+s e_{x}, u_{h}+s e_{u} ; z_{h}+s e_{z}, e\right) d s
\end{aligned}
$$

where $e=\left(e_{x}, e_{u}, e_{z}\right) \in \dot{W} \times U \times V$. Using the third equation in (2.3) and the third equation in (2.5) we get

$$
\begin{aligned}
\mathscr{J}(x, u)-\mathscr{J}\left(x_{h}, u_{h}\right)= & \mathscr{L}(x, u ; z)-\mathscr{F}(x, u ; z)-\mathscr{L}\left(x_{h}, u_{h} ; z_{h}\right)+\mathscr{F}\left(x_{h}, u_{h} ; z_{h}\right) \\
= & \mathscr{L}(x, u ; z)-\mathscr{L}\left(x_{h}, u_{h} ; z_{h}\right) \\
= & \mathscr{L}^{\prime}\left(x, x_{h}, u, u_{h} ; z, z_{h}, e\right)+\frac{1}{2} \mathscr{L}^{\prime}\left(x_{h}, u_{h} ; z_{h}, e\right) \\
& -\frac{1}{2} \mathscr{L}^{\prime}\left(x_{h}, u_{h} ; z_{h}, e\right)-\frac{1}{2} \mathscr{L}^{\prime}(x, u ; z, e),
\end{aligned}
$$

where the last term is zero in view of (2.2). The last two terms are equal to an approximation of the first term by the trapezoidal rule. Hence, with $R$ denoting the remainder in this approximation,

$$
\begin{aligned}
\mathscr{J}(x, u)-\mathscr{J}\left(x_{h}, u_{h}\right) & =\frac{1}{2} \mathscr{L}^{\prime}\left(x_{h}, u_{h} ; z_{h}, e\right)+R \\
& =\frac{1}{2} \mathscr{L}^{\prime}\left(x_{h}, u_{h} ; z_{h}, x-x_{h}, u-u_{h}, z-z_{h}\right)+R \\
& =\frac{1}{2} \mathscr{L}^{\prime}\left(x_{h}, u_{h} ; z_{h}, x-\tilde{x}_{h}, u-\tilde{u}_{h}, z-\tilde{z}_{h}\right)+R .
\end{aligned}
$$

Here we used the orthogonality property (2.4) to replace $\left(x_{h}, u_{h}, z_{h}\right)$ by an arbitrary $\left(\tilde{x}_{h}, \tilde{u}_{h}, \tilde{x}_{h}\right) \in \tilde{W}_{h} \times U_{h} \times V_{h}$. By expanding $\mathscr{L}^{\prime}$ in terms of partial derivatives we then obtain

$$
\begin{aligned}
\mathscr{J}(x, u)-\mathscr{J}\left(x_{h}, u_{h}\right)= & \frac{1}{2}\left(\mathscr{J}_{x}^{\prime}\left(x_{h}, u_{h} ; x-\tilde{x}_{h}\right)+\mathscr{F}_{x}^{\prime}\left(x_{h}, u_{h} ; z_{h}, x-\tilde{x}_{h}\right)\right) \\
& +\frac{1}{2}\left(\mathscr{J}_{u}^{\prime}\left(x_{h}, u_{h} ; u-\tilde{u}_{h}\right)+\mathscr{F}_{u}^{\prime}\left(x_{h}, u_{h} ; z_{h}, u-\tilde{u}_{h}\right)\right) \\
& +\frac{1}{2} \mathscr{F}\left(x_{h}, u_{h} ; z-\tilde{z}_{h}\right)+R \\
= & \frac{1}{2} \rho_{x}+\frac{1}{2} \rho_{u}+\frac{1}{2} \rho_{z}+R .
\end{aligned}
$$

The remainder term $R$ is

$$
\begin{aligned}
R= & \overline{\mathscr{L}}^{\prime}\left(x, x_{h}, u, u_{h} ; z, z_{h}, e\right)-\frac{1}{2} \mathscr{L}^{\prime}\left(x_{h}, u_{h} ; z_{h}, e\right)-\frac{1}{2} \mathscr{L}^{\prime}(x, u ; z, e) \\
= & \frac{1}{2} \int_{0}^{1} \mathscr{L}^{\prime \prime \prime}\left(x_{h}+s e_{x}, u_{h}+s e_{u} ; z_{h}+s e_{z}, e, e, e\right) s(s-1) d s \\
= & \frac{1}{2} \int_{0}^{1}\left(J^{\prime \prime \prime}\left(x_{h}+s e_{x}, u_{h}+s e_{u} ; e, e, e\right)\right. \\
& \left.+\mathscr{F}^{\prime \prime \prime}\left(x_{h}+s e_{x}, u_{h}+s e_{u} ; z_{h}+s e_{z}, e, e, e\right)\right) s(s-1) d s
\end{aligned}
$$




\section{An optimal control problem}

We consider optimal control problems of the form

$$
\begin{array}{ll}
\text { minimize } & l(x(0), x(T))+\int_{0}^{T} L(x(t), u(t)) d t, \\
\text { subject to } & \dot{x}(t)=f(x(t), u(t)), \quad 0<t<T, \\
& I_{0} x(0)=x_{0}, \quad I_{T} x(T)=x_{T} .
\end{array}
$$

Here

$$
\begin{aligned}
& l: \mathbb{R}^{d} \times \mathbb{R}^{d} \rightarrow \mathbb{R}, \\
& L: \mathbb{R}^{d} \times \mathbb{R}^{m} \rightarrow \mathbb{R}, \\
& f: \mathbb{R}^{d} \times \mathbb{R}^{m} \rightarrow \mathbb{R}^{d},
\end{aligned}
$$

are smooth functions, $I_{0}, I_{T} \in \mathbb{R}^{d \times d}$ are $d \times d$ matrices, and $x_{0} \in R\left(I_{0}\right), x_{T} \in R\left(I_{T}\right)$, where $R(A)$ denotes the range of a matrix $A$.

In order to put this into the abstract framework of the previous section, we need to introduce function spaces $W, \dot{W}, \tilde{W}, V, U$ and functionals $\mathscr{J}$ and $\mathscr{F}$. The spaces must accommodate both the continuous functions $x, z, u$ and the corresponding finite element functions. It is therefore convenient to begin by defining the finite element spaces.

We define a mesh $0=t_{0}<t_{1}<t_{2}<\ldots<t_{N}=T$, with steps $h_{n}=t_{n}-t_{n-1}$ and intervals $I_{n}=\left(t_{n-1}, t_{n}\right)$. Let $q \geq 0$ and let $P^{q}$ denote the polynomials of degree $\leq q$. We introduce the spaces

$$
\begin{aligned}
W_{h} & =\mathbb{R}^{d} \times\left\{w:\left.w\right|_{I_{n}} \in P^{q}\left(I_{n}, \mathbb{R}^{d}\right), n=1, \ldots, N\right\} \times \mathbb{R}^{d}, \\
\dot{W}_{h} & =R\left(I-I_{0}\right) \times\left\{w:\left.w\right|_{I_{n}} \in P^{q}\left(I_{n}, \mathbb{R}^{d}\right), n=1, \ldots, N\right\} \times R\left(I-I_{T}\right) \\
& =\left\{w \in W_{h}: I_{0} w_{0}^{-}=0, I_{T} w_{N}^{+}=0\right\},
\end{aligned}
$$

of (vector-valued) discontinuous piecewise polynomial functions of degree $\leq q$ and the space

$$
V_{h}=\left\{v \in C\left([0, T], \mathbb{R}^{d}\right):\left.v\right|_{I_{n}} \in P^{q+1}\left(I_{n}, \mathbb{R}^{d}\right)\right\},
$$

of continuous piecewise polynomial functions of degree $\leq q+1$. For $w \in W_{h}$ we use the notations $w_{n}^{ \pm}=\lim _{t \rightarrow t_{n}^{ \pm}} w(t)$ for the one-sided limits at $t_{n}$ and $[w]_{n}=w_{n}^{+}-w_{n}^{-}$, for the jump at $t_{n}$. For $v \in V_{h}$ we write $v_{n}=v\left(t_{n}\right)$. The two factors $\mathbb{R}^{d}$ in $W_{h}$ contain the boundary values $w_{0}^{-}$and $w_{N}^{+}$. We also select $\hat{x} \in W_{h}$ such that

$$
I_{0} \hat{x}_{0}^{-}=x_{0}, \quad I_{T} \hat{x}_{N}^{+}=x_{T},
$$

where $x_{0}, x_{T}$ are the boundary values in (3.1), and define the affine space

$$
\begin{aligned}
\tilde{W}_{h} & =\hat{x}+\dot{W}_{h}=\left\{w \in W_{h}: w-\hat{x} \in \dot{W}_{h}\right\} \\
& =\left\{w \in W_{h}: I_{0} w_{0}^{-}=x_{0}, I_{T} w_{N}^{+}=x_{T}\right\} .
\end{aligned}
$$


Finally, we define

$$
U_{h}=\left\{v \in C\left([0, T], \mathbb{R}^{m}\right):\left.v\right|_{I_{n}} \in P^{q+1}\left(I_{n}, \mathbb{R}^{m}\right)\right\} .
$$

Note that

$$
\begin{aligned}
\operatorname{dim}\left(W_{h}\right) & =(N(q+1)+2) d, \\
\operatorname{dim}\left(\dot{W}_{h}\right) & =(N(q+1)+2) d-d_{0}-d_{T}, \\
\operatorname{dim}\left(V_{h}\right) & =(N(q+1)+1) d, \\
\operatorname{dim}\left(U_{h}\right) & =(N(q+1)+1) m,
\end{aligned}
$$

where $d_{0}=\operatorname{rank}\left(I_{0}\right), d_{T}=\operatorname{rank}\left(I_{T}\right)$.

We now define the function spaces

$$
\begin{aligned}
W & =\mathbb{R}^{d} \times\left\{w:\left.w\right|_{I_{n}} \in H^{1}\left(I_{n}, \mathbb{R}^{d}\right), n=1, \ldots, N\right\} \times \mathbb{R}^{d}, \\
\dot{W} & =R\left(I-I_{0}\right) \times\left\{w:\left.w\right|_{I_{n}} \in H^{1}\left(I_{n}, \mathbb{R}^{d}\right), n=1, \ldots, N\right\} \times R\left(I-I_{T}\right) \\
& =\left\{w \in W: I_{0} w_{0}^{-}=0, I_{T} w_{N}^{+}=0\right\}, \\
\tilde{W} & =\hat{x}+\dot{W}=\{w \in W: w-\hat{x} \in \dot{W}\} \\
& =\left\{w \in W: I_{0} w_{0}^{-}=x_{0}, I_{T} w_{N}^{+}=x_{T}\right\}, \\
V & =H^{1}\left((0, T), \mathbb{R}^{d}\right), \\
U & =H^{1}\left((0, T), \mathbb{R}^{m}\right) .
\end{aligned}
$$

The spaces are equipped with the maximum norm. Note that, by Sobolev's inequality, functions in $W, \dot{W}$ are continuous on each interval $I_{n}$ with one-sided limits at the endpoints, and functions in $V, U$ are continuous on $[0, T]$. Boundary values are accommodated in $W$ in the same way as in $W_{h}$; of course, if $w \in W$ happens to be continuous, then $w_{0}^{-}=w_{0}^{+}=w(0)$ and $w_{N}^{-}=w_{N}^{+}=w(T)$ are the usual boundary values. The function spaces have been constructed so that $W_{h} \subset W, \dot{W}_{h} \subset \dot{W}, \tilde{W}_{h} \subset \tilde{W}$, $V_{h} \subset V$, and $U_{h} \subset U$.

The functional to be minimized is

$$
\mathscr{J}(w, u)=l\left(w_{0}^{-}, w_{N}^{+}\right)+\int_{0}^{T} L(w, u) d t, \quad(w, u) \in W \times U,
$$

and, for the weak formulation of the state equation, we define the functional

$$
\mathscr{F}(w, u ; v)=\sum_{n=1}^{N} \int_{I_{n}}(\dot{w}-f(w, u), v) d t+\sum_{n=0}^{N}\left([w]_{n}, v_{n}\right), \quad(w, u, v) \in W \times U \times V .
$$

Here and below, $(\cdot, \cdot)$ denotes the scalar product in $\mathbb{R}^{d}$ or $\mathbb{R}^{m}$. If $x$ is a smooth function which satisfies the state equation in (3.1), then it also satisfies the weak problem: find $x \in \tilde{W}$ such that

$$
\mathscr{F}(x, u ; \varphi)=0, \quad \forall \varphi \in V .
$$


Here we used the fact that $x_{0}^{-}=x(0), x_{N}^{+}=x(T),[x]_{n}=0$, because $x$ is continuous.

We now find it convenient to change the notation for partial derivatives. For a scalar-valued function

$$
g: \mathbb{R}^{d} \times \mathbb{R}^{m} \rightarrow \mathbb{R}
$$

we denote by $g_{i}^{\prime}(x, u)$ the partial derivative with respect to the $i^{\text {th }}$ variable. It is a linear operator $\mathbb{R}^{d} \rightarrow \mathbb{R}$ for $i=1$ and $\mathbb{R}^{m} \rightarrow \mathbb{R}$ for $i=2$, which we may identify with a vector, so that

$$
g_{1}^{\prime}(x, u) y=\left(y, g_{1}^{\prime}(x, u)\right), y \in \mathbb{R}^{d}, \quad g_{2}^{\prime}(x, u) y=\left(y, g_{2}^{\prime}(x, u)\right), y \in \mathbb{R}^{m} .
$$

For a vector-valued function

$$
f: \mathbb{R}^{d} \times \mathbb{R}^{m} \rightarrow \mathbb{R}^{d}
$$

the partial derivatives are linear operators $f_{1}^{\prime}(x, u): \mathbb{R}^{d} \rightarrow \mathbb{R}^{d}$ and $f_{2}^{\prime}(x, u): \mathbb{R}^{m} \rightarrow \mathbb{R}^{d}$, which we identify with matrices $f_{1}^{\prime}(x, u) \in \mathbb{R}^{d \times d}$ and $f_{2}^{\prime}(x, u) \in \mathbb{R}^{m \times d}$.

Integration by parts gives,

$$
\begin{aligned}
& \mathscr{F}_{1}^{\prime}(w, u ; v, \varphi) \\
& =\sum_{n=1}^{N} \int_{I_{n}}\left(\dot{\varphi}-f_{1}^{\prime}(w, u) \varphi, v\right) d t+\sum_{n=0}^{N}\left([\varphi]_{n}, v_{n}\right) \\
& =\sum_{n=1}^{N} \int_{I_{n}}\left(\varphi,-\dot{v}-f_{1}^{\prime}(w, u)^{*} v\right) d t+\left(\varphi_{N}^{+}, v_{N}\right)-\left(\varphi_{0}^{-}, v_{0}\right), \\
& \quad \forall(w, u, v, \varphi) \in W \times U \times V \times \dot{W} .
\end{aligned}
$$

The Lagrange functional is

$$
\mathscr{L}(x, u ; z)=\mathscr{J}(x, u)+\mathscr{F}(x, u ; z), \quad(w, u, z) \in W \times U \times V .
$$

The necessary condition for optimality is that $(x, u, z) \in \tilde{W} \times U \times V$ and

$$
\mathscr{L}^{\prime}(x, u ; z, \varphi)=0, \quad \forall \varphi \in \dot{W} \times U \times V,
$$

which yields

$$
\begin{array}{rlrl}
\mathscr{L}_{1}^{\prime}\left(x, u ; z, \varphi_{x}\right)=\mathscr{J}_{1}^{\prime}\left(x, u ; \varphi_{x}\right)+\mathscr{F}_{1}^{\prime}\left(x, u ; z, \varphi_{x}\right) & =0, & & \forall \varphi_{x} \in \dot{W}, \\
\mathscr{L}_{2}^{\prime}\left(x, u ; z, \varphi_{u}\right)=\mathscr{J}_{2}^{\prime}\left(x, u ; \varphi_{u}\right)+\mathscr{F}_{2}^{\prime}\left(x, u ; z, \varphi_{u}\right)=0, & \forall \varphi_{u} \in U, \\
\mathscr{L}_{3}^{\prime}\left(x, u ; z, \varphi_{z}\right)=0+\mathscr{F}\left(x, u ; \varphi_{z}\right)=0, & \forall \varphi_{z} \in V .
\end{array}
$$

The first equation in (3.6) is, in view of the second form of $\mathscr{F}_{1}^{\prime}$ in (3.4),

$$
\begin{aligned}
& \sum_{n=1}^{N} \int_{I_{n}}\left(\varphi, L_{1}^{\prime}(x, u)-\dot{z}-f_{1}^{\prime}(x, u)^{*} z\right) d t \\
& \quad+\left(\varphi_{N}^{+}, l_{2}^{\prime}\left(x_{0}^{-}, x_{N}^{+}\right)+z_{N}\right)+\left(\varphi_{0}^{-}, l_{1}^{\prime}\left(x_{0}^{-}, x_{N}^{+}\right)-z_{0}\right)=0, \quad \forall \varphi \in \dot{W} .
\end{aligned}
$$


Assuming that $x, \dot{z}, \varphi$ are continuous, we may identify the strong form of this equation:

$$
\begin{aligned}
& \dot{z}+f_{1}^{\prime}(x, u)^{*} z-L_{1}^{\prime}(x, u)=0, \quad 0<t<T, \\
& \left(I-I_{0}\right)\left(z(0)-l_{1}^{\prime}(x(0), x(T))\right)=0, \\
& \left(I-I_{T}\right)\left(z(T)+l_{2}^{\prime}(x(0), x(T))\right)=0,
\end{aligned}
$$

which is the linearized adjoint equation to the state equation in $(3.1)$. Note the complementary boundary conditions.

The second equation in $(3.6)$ is

$$
\int_{0}^{T}\left(\varphi, L_{2}^{\prime}(x, u)-f_{2}^{\prime}(x, u)^{*} z\right) d t=0, \quad \forall \varphi \in U
$$

or, in strong form,

$$
L_{2}^{\prime}(x, u)-f_{2}^{\prime}(x, u)^{*} z=0, \quad 0<t<T .
$$

This a non-linear algebraic equation for $u$. The third equation is the same as (3.3).

We next formulate the finite element approximation of these equations. Find $\left(x_{h}, u_{h}, z_{h}\right) \in \tilde{W}_{h} \times U_{h} \times V_{h}$ such that

$$
\mathscr{L}^{\prime}\left(x_{h}, u_{h} ; z_{h}, \varphi\right)=0, \quad \forall \varphi \in \dot{W}_{h} \times U_{h} \times V_{h}
$$

which means that we want to determine $\left(x_{h}, u_{h}, z_{h}\right) \in W_{h} \times U_{h} \times V_{h}$ such that

$$
\begin{aligned}
& \sum_{n=1}^{N} \int_{I_{n}}\left(\varphi, L_{1}^{\prime}\left(x_{h}, u_{h}\right)-\dot{z}_{h}-f_{1}^{\prime}\left(x_{h}, u_{h}\right)^{*} z_{h}\right) d t \\
& \quad+\left(\varphi_{N}^{+}, l_{2}^{\prime}\left(x_{h, 0}^{-}, x_{h, N}^{+}\right)+z_{h, N}\right)+\left(\varphi_{0}^{-}, l_{1}^{\prime}\left(x_{h, 0}^{-}, x_{h, N}^{+}\right)-z_{h, 0}\right)=0, \\
& \quad \forall \varphi \in \dot{W}_{h}, \\
& \int_{0}^{T}\left(\varphi, L_{2}^{\prime}\left(x_{h}, u_{h}\right)-f_{2}^{\prime}\left(x_{h}, u_{h}\right)^{*} z_{h}\right) d t=0, \quad \forall \varphi \in U_{h}, \\
& \left\{\begin{array}{l}
I_{0} x_{h, 0}^{-}=x_{0}, \quad I_{T} x_{h, N}^{+}=x_{T}, \\
\sum_{n=1}^{N} \int_{I_{n}}\left(\dot{x}_{h}-f\left(x_{h}, u_{h}\right), \varphi\right) d t+\sum_{n=0}^{N}\left(\left[x_{h}\right]_{n}, \varphi_{n}\right)=0, \quad \forall \varphi \in V_{h} .
\end{array}\right.
\end{aligned}
$$

Using (3.2) we easily verify that these are $N(q+1)(2 d+m)+3 d+m$ algebraic equations in equally many unknowns.

Since $\varphi_{0}^{-}$and $\varphi_{N}^{+}$can be chosen arbitrarily in $R\left(I-I_{0}\right)$ and $R\left(I-I_{T}\right)$, respectively, we see that (3.8) implies

$$
\begin{aligned}
& \left(I-I_{0}\right)^{*}\left(l_{1}^{\prime}\left(x_{h, 0}^{-}, x_{h, N}^{+}\right)-z_{h, 0}\right)=0 \\
& \left(I-I_{T}\right)^{*}\left(l_{2}^{\prime}\left(x_{h, 0}^{-}, x_{h, N}^{+}\right)+z_{h, N}\right)=0 .
\end{aligned}
$$

The a posteriori error representation formula follows from Theorem 2.2 . 
Corollary 3.1 Let $(x, u, z) \in \tilde{W} \times U \times V$ and $\left(x_{h}, u_{h}, z_{h}\right) \in \tilde{W}_{h} \times U_{h} \times V_{h}$ be solutions of (3.5) and (3.7), respectively. Then

$$
\mathscr{J}(x, u)-\mathscr{J}\left(x_{h}, u_{h}\right)=\frac{1}{2} \rho_{x}+\frac{1}{2} \rho_{z}+\frac{1}{2} \rho_{u}+R,
$$

with the residuals $\rho_{x}, \rho_{z}$, and $\rho_{u}$ defined as

$$
\begin{aligned}
& \rho_{x}=\sum_{n=1}^{N} \int_{I_{n}}\left(x-\tilde{x}_{h}, L_{1}^{\prime}\left(x_{h}, u_{h}\right)-\dot{z}_{h}-f_{1}^{\prime}\left(x_{h}, u_{h}\right)^{*} z_{h}\right) d t, \\
& \rho_{u}=\int_{0}^{T}\left(u-\tilde{u}_{h}, L_{2}^{\prime}\left(x_{h}, u_{h}\right)-f_{2}^{\prime}\left(x_{h}, u_{h}\right)^{*} z_{h}\right) d t, \\
& \rho_{z}=\sum_{n=1}^{N} \int_{I_{n}}\left(\dot{x}_{h}-f\left(x_{h}, u_{h}\right), z-\tilde{z}_{h}\right) d t+\sum_{n=0}^{N}\left(\left[x_{h}\right]_{n}, z_{n}-\tilde{z}_{h, n}\right),
\end{aligned}
$$

where $\left(\tilde{x}_{h}, \tilde{u}_{h}, \tilde{z}_{h}\right) \in \tilde{W}_{h} \times U_{h} \times V_{h}$ is arbitrary, and the remainder $R$ is given by (2.6).

Proof From Theorem 2.2 we have

$$
\begin{aligned}
\rho_{x}= & \sum_{n=1}^{N} \int_{I_{n}}\left(x-\tilde{x}_{h}, L_{1}^{\prime}\left(x_{h}, u_{h}\right)-\dot{z}_{h}-f_{1}^{\prime}\left(x_{h}, u_{h}\right)^{*} z_{h}\right) d t \\
& +\left(x_{N}^{+}-\tilde{x}_{h, N}^{+}, l_{2}^{\prime}\left(x_{h, 0}^{-}, x_{h, N}^{+}\right)+z_{h, N}\right) \\
& +\left(x_{0}^{-}-\tilde{x}_{h, 0}^{-}, l_{1}^{\prime}\left(x_{h, 0}^{-}, x_{h, N}^{+}\right)-z_{h, 0}\right) .
\end{aligned}
$$

Using (3.11) and $I_{0}\left(x_{0}^{-}-\tilde{x}_{h, 0}^{-}\right)=0, I_{T}\left(x_{N}^{+}-\tilde{x}_{h, N}^{+}\right)=0$, we find

$$
\begin{aligned}
& \left(x_{N}^{+}-\tilde{x}_{h, N}^{+}, l_{2}^{\prime}\left(x_{h, 0}^{-}, x_{h, N}^{+}\right)+z_{h, N}\right)=0, \\
& \left(x_{0}^{-}-\tilde{x}_{h, 0}^{-}, l_{1}^{\prime}\left(x_{h, 0}^{-}, x_{h, N}^{+}\right)-z_{h, 0}\right)=0,
\end{aligned}
$$

and we obtain the desired form of $\rho_{x}$. The other residuals, $\rho_{u}$ and $\rho_{z}$, follow directly from Theorem 2.2 .

\section{A quadratic/linear optimal control problem}

4.1 The continuous problem

In this section we specialize to the case when the functional to be minimized is quadratic and the state equation is linear. The reason for studying this simplified case is that it makes the formulation and implementation of an adaptive algorithm easier. It is also true that many models are formulated as quadratic/linear problems. We use the notation $\|v\|_{S}^{2}=(v, S v)$, where $(\cdot, \cdot)$ is the scalar product and $S$ is a symmetric, positive semidefinite matrix. The problem then reads

$$
\begin{aligned}
& \text { minimize } \quad \mathscr{J}(x, u)=\left\|x(0)-\bar{x}_{0}\right\|_{S_{0}}^{2}+\left\|x(T)-\bar{x}_{T}\right\|_{S_{T}}^{2} \\
& +\int_{0}^{T}\left(\|u-\bar{u}\|_{R}^{2}+\|x-\bar{x}\|_{Q}^{2}\right) d t, \\
& \text { subject to } \quad \dot{x}=A(t) x+B(t) u, \quad 0<t<T, \\
& I_{0} x(0)=x_{0}, \quad I_{T} x(T)=x_{T},
\end{aligned}
$$


where, for each $t, Q(t), S_{0}, S_{T} \in \mathbb{R}^{d \times d}$ are symmetric positive semidefinite matrices, $R(t) \in \mathbb{R}^{m \times m}$ is a symmetric positive definite matrix, and $A(t) \in \mathbb{R}^{d \times d}$ and $B(t) \in$ $\mathbb{R}^{d \times m}$ are matrices. The matrices $I_{0}, I_{T} \in \mathbb{R}^{d \times d}, x_{0}, x_{T}, \bar{x}_{0}, \bar{x}_{T}, \bar{x}(t)$, and $\bar{u}(t)$ are given.

Since we now have

$$
\begin{array}{ll}
f(x, u)=A x+B u, & \\
f_{1}^{\prime}(x, u)=A, & f_{2}^{\prime}(x, u)=B, \\
L_{1}^{\prime}(x, u)=2 Q(x-\bar{x}), & L_{2}^{\prime}(x, u)=2 R(u-\bar{u}), \\
l_{1}^{\prime}\left(x_{0}^{-}, x_{N}^{+}\right)=2 S_{0}\left(x_{0}^{-}-\bar{x}_{0}\right), & l_{2}^{\prime}\left(x_{0}^{-}, x_{N}^{+}\right)=2 S_{T}\left(x_{N}^{+}-\bar{x}_{T}\right),
\end{array}
$$

the equation (3.5) is now to find $(x, u, z) \in \tilde{W} \times U \times V$ such that

$$
\begin{aligned}
& \int_{0}^{T}\left(\varphi_{x}, 2 Q(x-\bar{x})-\dot{z}-A^{*} z\right) d t \\
& \quad+\left(\varphi_{x, 0}^{-}, 2 S_{0}\left(x_{0}^{-}-\bar{x}_{0}\right)-z_{0}\right) \\
& \quad+\left(\varphi_{x, N}^{+}, 2 S_{T}\left(x_{N}^{+}-\bar{x}_{T}\right)+z_{N}\right)=0, \quad \forall \varphi_{x} \in \dot{W}, \\
& \int_{0}^{T}\left(\varphi_{u}, 2 R(u-\bar{u})-B^{*} z\right) d t=0, \quad \forall \varphi_{u} \in U, \\
& \int_{0}^{T}\left(\dot{x}-A x-B u, \varphi_{z}\right) d t=0, \quad \forall \varphi_{z} \in V .
\end{aligned}
$$

\subsection{The finite element method}

Let the finite element spaces be as in Section 3. We discretize the state equation (4.4) by a discontinuous Galerkin method with $W_{h}$ as trial space and $V_{h}$ as test space: Seek $x_{h} \in W_{h}$ which fulfils

$$
\begin{aligned}
& I_{0} x_{h, 0}^{-}=x_{0}, \quad I_{T} x_{h, N}^{+}=x_{T}, \\
& \int_{0}^{T}\left(\dot{x}_{h}-A x_{h}-B u_{h}, \varphi\right) d t+\sum_{n=0}^{N}\left(\left[x_{h}\right]_{n}, \varphi_{n}\right)=0, \quad \forall \varphi \in V_{h} .
\end{aligned}
$$

The dual equation (4.2) is discretized by the continuous Galerkin method: Seek $z_{h} \in$ $V_{h}$ which fulfils

$$
\begin{aligned}
& \int_{0}^{T}\left(\varphi, 2 Q\left(x_{h}-\bar{x}\right)-\dot{z}_{h}-A^{*} z_{h}\right) d t \\
& \quad+\left(\varphi_{0}^{-}, 2 S_{0}\left(x_{h, 0}^{-}-\bar{x}_{0}\right)-z_{h, 0}\right) \\
& \quad+\left(\varphi_{N}^{+}, 2 S_{T}\left(x_{h, N}^{+}-\bar{x}_{T}\right)+z_{h, N}\right)=0, \quad \forall \varphi \in \dot{W}_{h},
\end{aligned}
$$

where we have used $V_{h}$ as trial space and $\dot{W}_{h}$ as test space. Since we can vary the boundary values in $\dot{W}_{h}$ separately in $R\left(I-I_{0}\right)$ and $R\left(I-I_{T}\right)$, the boundary conditions become

$$
\begin{aligned}
& \left(I-I_{0}\right)^{*}\left(z_{h, 0}-2 S_{0}\left(x_{h, 0}^{-}-\bar{x}_{0}\right)\right)=0 \\
& \left(I-I_{T}\right)^{*}\left(z_{h, N}+2 S_{T}\left(x_{h, N}^{+}-\bar{x}_{T}\right)\right)=0
\end{aligned}
$$


Equation (4.3) for the controls is discretized by a continuous Galerkin method: Seek $u_{h} \in U_{h}$

$$
\int_{0}^{T}\left(\varphi, 2 R\left(u_{h}-\bar{u}\right)-B^{*} z_{h}\right) d t=0, \quad \forall \varphi_{u} \in U_{h} .
$$

We now have three sets of linear algebraic equations which must be solved simultaneously in order to obtain the approximate solution $\left(x_{h}, u_{h}, z_{h}\right)$.

\subsection{The error estimate}

We begin by repeating the error representation formula from Corollary 3.1 in the context of the linear/quadratic optimal control problem.

Corollary 4.1 Let $(x, u, z) \in \tilde{W} \times U \times V$ and $\left(x_{h}, u_{h}, z_{h}\right) \in \tilde{W}_{h} \times U_{h} \times V_{h}$ be solutions of (4.2)-(4.4) and (4.5)-(4.7), respectively. Then

$$
\mathscr{J}(x, u)-\mathscr{J}\left(x_{h}, u_{h}\right)=\frac{1}{2} \rho_{x}+\frac{1}{2} \rho_{z}+\frac{1}{2} \rho_{u},
$$

with $\rho_{x}, \rho_{z}$, and $\rho_{u}$ defined as

$$
\begin{aligned}
& \rho_{x}=\int_{0}^{T}\left(x-\tilde{x}_{h}, 2 Q\left(x_{h}-\bar{x}\right)-\dot{z}_{h}-A^{*} z_{h}\right) d t, \\
& \rho_{u}=\int_{0}^{T}\left(u-\tilde{u}_{h}, 2 R\left(u_{h}-\bar{u}\right)-B^{*} z_{h}\right) d t, \\
& \rho_{z}=\int_{0}^{T}\left(\dot{x}_{h}-A x_{h}-B u_{h}, z-\tilde{z}_{h}\right) d t+\sum_{n=0}^{N}\left(\left[x_{h}\right]_{n}, z_{n}-\tilde{z}_{h, n}\right),
\end{aligned}
$$

where $\left(\tilde{x}_{h}, \tilde{u}_{h}, \tilde{z}_{h}\right) \in \tilde{W}_{h} \times U_{h} \times V_{h}$ is arbitrary.

Proof The proof is a straightforward calculation using Corollary 3.1. The remainder $R$ is zero in this case, since we have a linear/quadratic problem and the remainder is the third derivative of the Lagrangian.

In the following theorem we derive an a posteriori error estimate from the error representation formula. We use the notation $\|f\|_{I_{n}}=\sup _{t \in I_{n}}\|f(t)\|$, where $\|\cdot\|$ denotes the Euclidean norm in $\mathbb{R}^{d}$ or $\mathbb{R}^{m}$.

Theorem 4.1 Let $(x, u, z) \in \tilde{W} \times U \times V$ and $\left(x_{h}, u_{h}, z_{h}\right) \in \tilde{W}_{h} \times U_{h} \times V_{h}$ be solutions of (4.2)-(4.4) and (4.5)-(4.7), respectively. Then

$$
\left|\mathscr{J}(x, u)-\mathscr{J}\left(x_{h}, u_{h}\right)\right| \leq \frac{1}{2} \sum_{n=1}^{N}\left(R_{n}^{z} \omega_{n}^{x}+R_{n}^{u} \omega_{n}^{u}+R_{n}^{x} \omega_{n}^{z}\right),
$$


where the residuals $R_{n}$ and weights $\omega_{n}$ are defined by (with $h_{0}=h_{N}=0$ )

$$
\begin{aligned}
R_{n}^{z}= & h_{n}\left\|2 Q\left(x_{h}-\bar{x}\right)-\dot{z}_{h}-A^{*} z_{h}\right\|_{I_{n}}, \\
R_{n}^{u}= & h_{n}\left\|2 R\left(u_{h}-\bar{u}\right)-B^{*} z_{h}\right\|_{I_{n}}, \\
R_{n}^{x}= & h_{n}\left\|\dot{x}_{h}-A x_{h}-B u_{h}\right\|_{I_{n}}+\frac{h_{n}}{h_{n}+h_{n+1}}\left\|\left[x_{h}\right]_{n}\right\| \\
& +\frac{h_{n}}{h_{n}+h_{n-1}}\left\|\left[x_{h}\right]_{n-1}\right\|,
\end{aligned}
$$

and, with arbitrary $\left(\tilde{x}_{h}, \tilde{u}_{h}, \tilde{z}_{h}\right) \in \tilde{W}_{h} \times U_{h} \times V_{h}$,

$$
\omega_{n}^{x}=\left\|x-\tilde{x}_{h}\right\|_{I_{n}}, \quad \omega_{n}^{u}=\left\|u-\tilde{u}_{h}\right\|_{I_{n}}, \quad \omega_{n}^{z}=\left\|z-\tilde{z}_{h}\right\|_{I_{n}} .
$$

Proof We estimate the three contributions to the error representation (4.8) separately. The first term is

$$
\begin{aligned}
\left|\rho_{x}\right| & \leq \sum_{n=1}^{N} \int_{I_{n}}\left\|x-\tilde{x}_{h}\right\|\left\|2 Q\left(x_{h}-\bar{x}\right)-\dot{z}_{h}-A^{*} z_{h}\right\| d t \\
& \leq \sum_{n=1}^{N}\left\|x-\tilde{x}_{h}\right\|_{I_{n}}\left\|2 Q\left(x_{h}-\bar{x}\right)-\dot{z}_{h}-A^{*} z_{h}\right\|_{I_{n}} h_{n}=\sum_{n=1}^{N} \omega_{n}^{x} R_{n}^{z} .
\end{aligned}
$$

Similarly, for the second term we have

$$
\left|\rho_{u}\right| \leq \sum_{n=1}^{N}\left\|u-\tilde{u}_{h}\right\|_{I_{n}}\left\|2 R\left(u_{h}-\bar{u}\right)-B^{*} z_{h}\right\|_{I_{n}} h_{n}=\sum_{n=1}^{N} \omega_{n}^{u} R_{n}^{u} .
$$

Finally,

$$
\begin{aligned}
\left|\rho_{z}\right| & \leq \sum_{n=1}^{N} \int_{I_{n}}\left\|\dot{x}_{h}-A x_{h}-B u_{h}\right\|\left\|z-\tilde{z}_{h}\right\| d t+\sum_{n=0}^{N}\left\|\left[x_{h}\right]_{n}\right\|\left\|z_{n}-\tilde{z}_{h, n}\right\| \\
& \leq \sum_{n=1}^{N}\left\|\dot{x}_{h}-A x_{h}-B u_{h}\right\|_{I_{n}}\left\|z-\tilde{z}_{h}\right\|_{I_{n}} h_{n}+\sum_{n=0}^{N}\left\|\left[x_{h}\right]_{n}\right\|\left\|z_{n}-\tilde{z}_{h, n}\right\| .
\end{aligned}
$$

Using the continuity of $z$ we have

$$
\left\|z_{n}-\tilde{z}_{h, n}\right\| \leq\left\|z-\tilde{z}_{h}\right\|_{I_{n}}, \quad\left\|z_{n}-\tilde{z}_{h, n}\right\| \leq\left\|z-\tilde{z}_{h}\right\|_{I_{n+1}}
$$

so that

$$
\begin{aligned}
\sum_{n=0}^{N}\left\|\left[x_{h}\right]_{n}\right\|\left\|z_{n}-\tilde{z}_{h, n}\right\| \\
=\sum_{n=1}^{N}\left(\frac{h_{n}}{h_{n}+h_{n+1}}\left\|\left[x_{h}\right]_{n}\right\|\left\|z_{n}-\tilde{z}_{h, n}\right\|\right. \\
\left.\quad+\frac{h_{n}}{h_{n}+h_{n-1}}\left\|\left[x_{h}\right]_{n-1}\right\|\left\|z_{n-1}-\tilde{z}_{h, n-1}\right\|\right) \\
\leq \sum_{n=1}^{N}\left(\frac{h_{n}}{h_{n}+h_{n+1}}\left\|\left[x_{h}\right]_{n}\right\|+\frac{h_{n}}{h_{n}+h_{n-1}}\left\|\left[x_{h}\right]_{n-1}\right\|\right)\|z-\tilde{z}\|_{I_{n}},
\end{aligned}
$$


where $h_{0}=h_{N}=0$. This yields

$$
\begin{aligned}
\left|\rho_{z}\right| \leq & \sum_{n=1}^{N}\left(h_{n}\left\|\dot{x}_{h}-A x_{h}-B u_{h}\right\|_{I_{n}}+\frac{h_{n}}{h_{n}+h_{n+1}}\left\|\left[x_{h}\right]_{n}\right\|\right. \\
& \left.+\frac{h_{n}}{h_{n}+h_{n-1}}\left\|\left[x_{h}\right]_{n-1}\right\|\right)\left\|z-\tilde{z}_{h}\right\|_{I_{n}}=\sum_{n=1}^{N} R_{n}^{x} \omega_{n}^{z} .
\end{aligned}
$$

We note that the error estimate does not introduce any additional adjoint equation. However, the weights depend on the exact solutions $x, u, z$ and approximations $\tilde{x}_{h}, \tilde{u}_{h}, \tilde{z}_{h}$ of them. In practice, we approximate the weights by computable quantities. For example, when $q=0$, by standard interpolation error estimates [4], we can find $\tilde{x}_{h}, \tilde{u}_{h}, \tilde{z}_{h}$ such that

$$
\begin{aligned}
\left\|x-\tilde{x}_{h}\right\|_{I_{n}} & \leq h_{n}\|\dot{x}\|_{I_{n}}, \\
\left\|u-\tilde{u}_{h}\right\|_{I_{n}} & \leq h_{n}^{2}\|\ddot{u}\|_{I_{n}}, \\
\left\|z-\tilde{z}_{h}\right\|_{I_{n}} & \leq h_{n}^{2}\|\ddot{z}\|_{I_{n}},
\end{aligned}
$$

where the derivatives are approximated by difference quotients of the discrete solutions. See also [1] for other approximations of the weights.

The above estimates of the weights indicate that the term $\rho_{z}$ in the error estimate is $O(h)$, while $\rho_{x}$ and $\rho_{u}$ are $O\left(h^{2}\right)$. We therefore present the following error estimate, where all terms are formally $O\left(h^{2}\right)$. For simplicity we assume that $A(t)=A$ and $Q(t)=Q$ are constant.

Theorem 4.2 Let $q=0$ and assume that $A(t)=A$ and $Q(t)=Q$ are constant. Then

$$
\begin{aligned}
\left|\mathscr{J}(x, u)-\mathscr{J}_{h}\left(x_{h}, u_{h}\right)\right| \leq & \sum_{n=1}^{N}\left(h_{n}^{3}\|\dot{x}\|_{I_{n}}\left\|2 Q \dot{\bar{x}}+A^{*} \dot{z}_{h}\right\|_{I_{n}}\right. \\
& +h_{n}^{3}\left\|A x_{h}+B u_{h}\right\|_{I_{n}}\|\ddot{z}\|_{I_{n}} \\
& \left.+h_{n}^{3}\left\|2 R\left(u_{h}-\bar{u}\right)-B^{*} z_{h}\right\|_{I_{n}}\|\ddot{u}\|_{I_{n}}\right) .
\end{aligned}
$$

Proof We choose $\tilde{z}_{h}=I_{h} z$ and $\tilde{u}_{h}=I_{h} u$ to be the standard piecewise linear nodal interpolators, and we choose $\tilde{x}_{h}=P_{h} x$ to be the orthogonal projection onto the piecewise constant functions. 
Then, using orthogonality, the fact that $z_{n}-\tilde{z}_{h, n}=0$, and the error estimates (4.11), in the error representation formula (4.8), we obtain

$$
\begin{aligned}
\mathscr{J}(x, u) & -\mathscr{J}\left(x_{h}, u_{h}\right) \\
= & \mid \sum_{n=1}^{N}\left(\int_{I_{n}}\left(\left(I-P_{h}\right) x,\left(I-P_{h}\right)\left(2 Q\left(x_{h}-\bar{x}\right)-\dot{z}_{h}-A^{*} z_{h}\right)\right) d t\right. \\
& +\int_{I_{n}}\left(\dot{x}_{h}-A x_{h}-B u_{h},\left(I-I_{h}\right) z\right) d t \\
& \left.+\int_{I_{n}}\left(\left(I-I_{h}\right) u, 2 R\left(u_{h}-\bar{u}\right)-B^{*} z_{h}\right) d t\right) \mid \\
\leq & \sum_{n=1}^{N}\left(h_{n}^{3}\|\dot{x}\|_{I_{n}}\left\|2 Q\left(\dot{x}_{h}-\dot{\bar{x}}\right)-\ddot{z}_{h}-A^{*} \dot{z}_{h}\right\|_{I_{n}}\right. \\
& +h_{n}^{3}\left\|\dot{x}_{h}-A x_{h}-B u_{h}\right\|_{I_{n}}\|\ddot{z}\|_{I_{n}} \\
& \left.+h_{n}^{3}\left\|2 R\left(u_{h}-\bar{u}\right)-B^{*} z_{h}\right\|_{I_{n}}\|\ddot{u}\|_{I_{n}}\right) .
\end{aligned}
$$

Since $\dot{x}_{h}=0$ and $\ddot{z}_{h}=0$ we obtain the desired estimate.

\subsection{An adaptive algorithm}

We have implemented an adaptive finite element method with $q=0$ based on the error estimate in the previous theorem, for the solution of the optimal control problem (4.1).

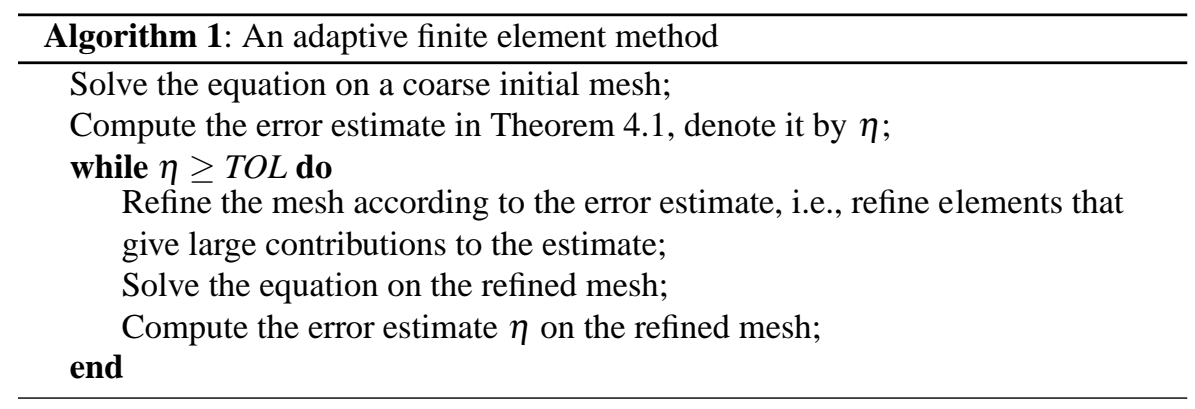

The refinement of the mesh is done according to the principle of equidistribution, that is, we want all intervals to give equally large contributions to the error estimate and we insert new nodes to fulfil this criterion. The implementation was done in Matlab. Numerical examples are given in the next section. The adaptivity leads to additional computational cost compared with a standard approach based on standard differential equation solvers and optimization procedures. The advantage of the finite element approach is the error control. Since we have not optimized the implementation we cannot present any comparison of the efficiency of our algorithm with other software. 


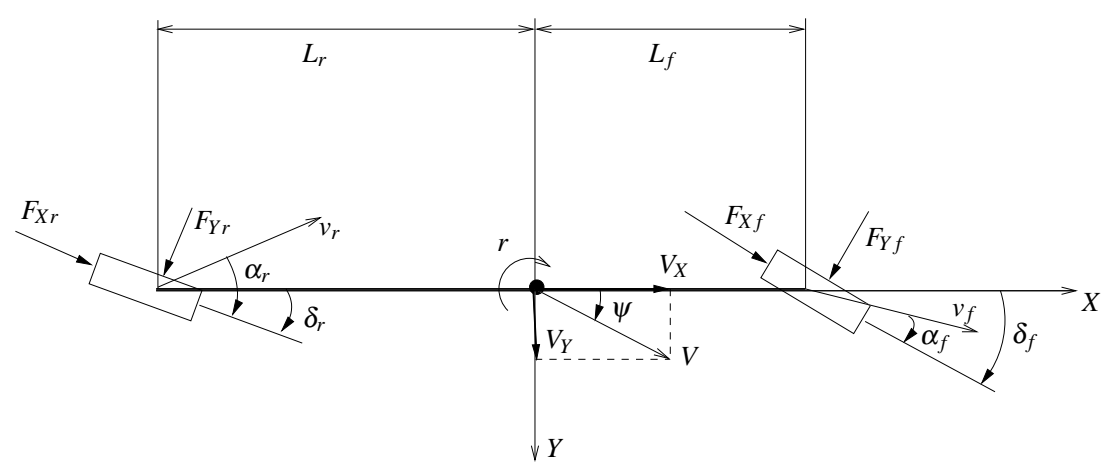

Fig. 5.1 The bicycle model, which is used to derive a model of the dynamics of a vehicle. The rectangles represent the wheels of the bicycle, and the dot marks the center of gravity around which the angular velocity is computed.

\section{Two numerical examples}

The adaptive finite element solver is tested on two quadratic/linear problems. They are both based on the so-called bicycle model from vehicle dynamics [8], see Figure 5.1. We study two manoeuvres, a single lane change and a single lane change with a light collision. The state variable,

$$
x=\left[\begin{array}{l}
x_{1} \\
x_{2} \\
x_{3} \\
x_{4} \\
x_{5} \\
x_{6}
\end{array}\right]=\left[\begin{array}{c}
V_{Y} \\
r \\
\psi \\
Y \\
\delta_{\mathrm{f}} \\
\delta_{\mathrm{r}}
\end{array}\right]
$$

consists of the lateral velocity $V_{Y}$, the angular velocity $r$, the heading angle $\psi$, the lateral position $Y$, and the front and rear steering angles $\delta_{\mathrm{f}}$ and $\delta_{\mathrm{r}}$. The longitudinal velocity $V_{X}$ is constant. The control variable $u=\left(u_{1}, u_{2}\right)$ consists of the inputs to the front and rear steering angles. The differential equations are

$$
\dot{x}=\left[\begin{array}{c}
\dot{V}_{Y} \\
\dot{r} \\
\dot{\psi} \\
\dot{Y} \\
\dot{\delta}_{\mathrm{f}} \\
\dot{\delta}_{\mathrm{r}}
\end{array}\right]=\left[\begin{array}{c}
a_{11} V_{Y}+a_{12} r+b_{\mathrm{f} 1} \delta_{\mathrm{f}}+b_{\mathrm{r} 1} \delta_{\mathrm{r}} \\
a_{21} V_{Y}+a_{22} r+b_{\mathrm{f} 2} \delta_{\mathrm{f}}+b_{\mathrm{r} 2} \delta_{\mathrm{r}} \\
r \\
V_{Y}+V_{X} \psi \\
-0.5 \delta_{\mathrm{f}}-0.5 u_{1} \\
-\delta_{\mathrm{r}}-u_{2}
\end{array}\right]=A x+B u .
$$

Our problem is of the form (4.1):

$$
\begin{array}{cl}
\text { minimize } & \mathscr{J}(x, u)=\int_{0}^{T}\left(\|u\|_{R}^{2}+\|x\|_{Q}^{2}\right) d t, \\
\text { subject to } & \dot{x}=A x+B u, \quad 0<t<T, \\
& I_{0} x(0)=x_{0}, \quad I_{T} x(T)=x_{T},
\end{array}
$$


where $I_{0}$ and $I_{T}$ are diagonal matrices. The coefficients $A, B, Q$, and $R$ can be found in the Appendix.

\subsection{Single lane change}

The velocity in the $X$-direction is $V_{X}=25 \mathrm{~m} / \mathrm{s}$ and the final time is $T=4 \mathrm{~s}$. We use the boundary conditions $x_{1}(0)=x_{2}(0)=x_{3}(0)=x_{5}(0)=x_{6}(0)=0, x_{4}(0)=10$, and $x_{1}(T)=x_{2}(T)=x_{3}(T)=x_{4}(T)=x_{5}(T)=x_{6}(T)=0$. This means that the dual variables have no boundary conditions. The problem describes a vehicle performing a lane change starting at $Y=10 \mathrm{~m}$ and ending at $Y=0 \mathrm{~m}$. We minimize the controls and all the states, but with different weights. The result is shown in Figure 5.2. Figure 5.2(a) shows the optimal track and Figures 5.2(b) and 5.2(c) show the optimal steering angles on the rear and front wheels. The adaptively refined mesh can be seen in Figure 5.2(d). The largest element is $6.25 \cdot 10^{-3}$ and the smallest one is of size $3.13 \cdot 10^{-3}$.

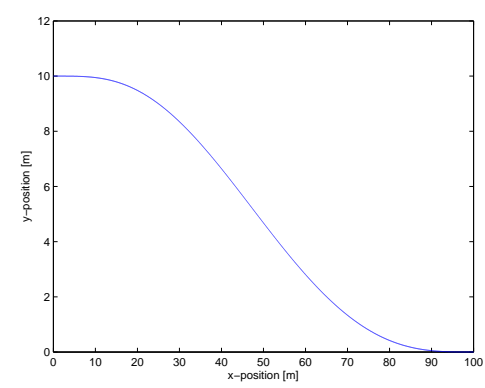

(a) Optimal track.

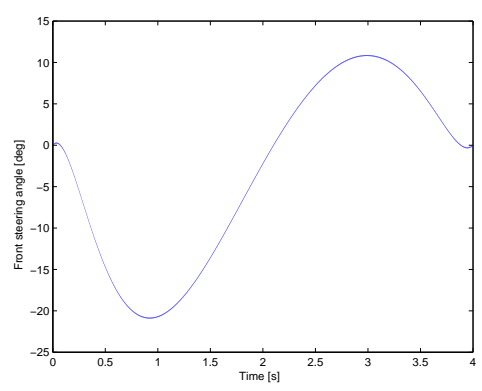

(c) Optimal steering angle, front

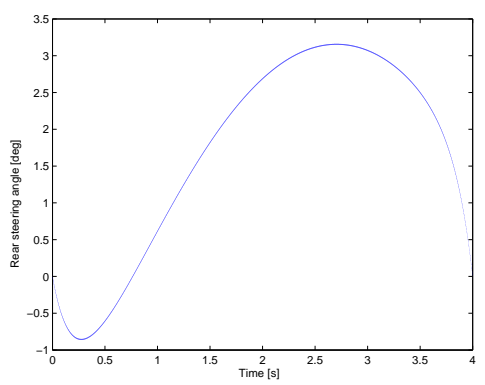

(b) Optimal steering angle, rear.

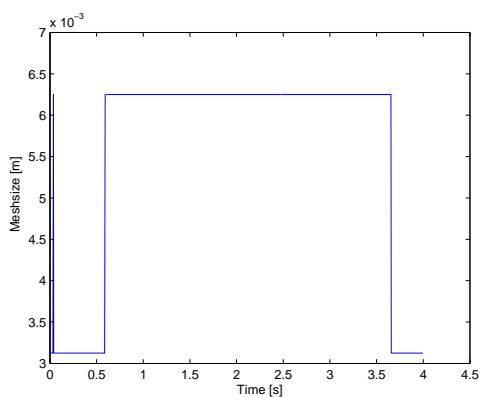

(d) Adaptively refined mesh.

Fig. 5.2 We see the optimal lane change manoeuvre and the optimal controls to perform this manoeuvre. In the last figure we see that the adaptive algorithm inserts nodes in the beginning and the end of the interval. 


\subsection{Lane change with collision}

This manoeuvre has the same boundary conditions and $X$-velocity as in the previous example, and $T=3 \mathrm{~s}$. However, when $t=0.5, x_{1}$ is momentarily increased by 2 and $x_{2}$ is increased by 0.1 . This can be described as a collision where the vehicle is subjected to an impulsive force and torque. This is introduced in order to test the adaptive solver on a more difficult problem than the previous one.

The result is shown in Figure 5.3. We see in Figure 5.3(d) that the solver refines the mesh mainly in the beginning of the manoeuvre and then inserts nodes around $t=0.5 \mathrm{~s}$ where the collision occurs. The largest element is of size $2.93 \cdot 10^{-2}$ and the smallest one is $1.87 \cdot 10^{-4}$.

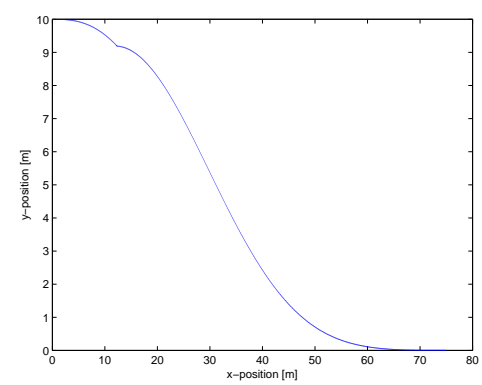

(a) Optimal track.

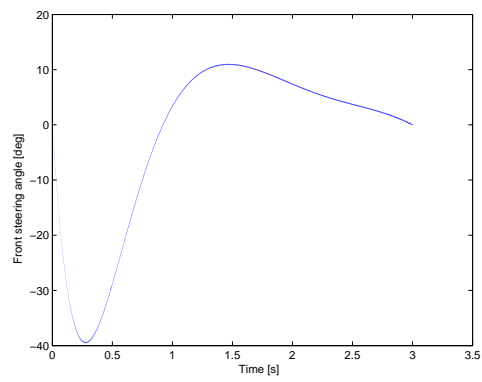

(c) Optimal steering angle, front.

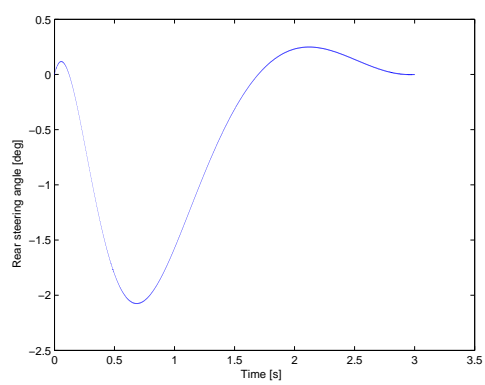

(b) Optimal steering angle, rear.

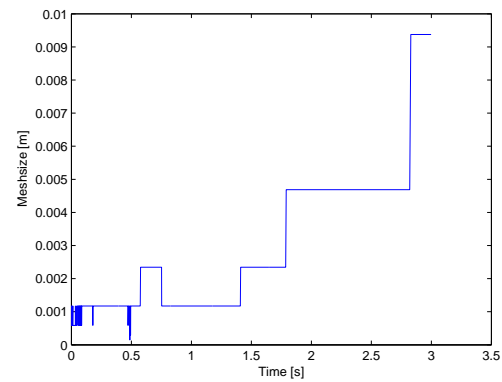

(d) Adaptively refined mesh.

Fig. 5.3 The optimal track and steering angles for the lane change manoeuvre where a collsion takes place during the lane change. Compared to the results in 5.2(d) we see that the adaptive solver inserts nodes around the time of the collision ( $t=0.5 \mathrm{~s}$ ).

Acknowledgements The authors would like thank Mathias Lidberg, Department of Applied Mechanics, Chalmers University of Technology for helpful discussions. 


\section{References}

1. Becker, R., Rannacher, R.: An optimal control approach to a posteriori error estimation in finite element methods. Acta Numer. 10, 1-102 (2001)

2. Betts, J.T.: Practical Methods for Optimal Control Using Nonlinear Programming. SIAM, Philadelphia (2001)

3. Bryson Jr, A.E., Ho, Y.: Applied Optimal Control. Hemisphere Publishing Corporation, Washington, D.C. (1975)

4. Eriksson, K., Estep, D., Hansbo, P., Johnson, C.: Computational Differential Equations. Cambridge University Press, Cambridge (1996)

5. Estep, D., Hodges, D.H., Warner, M.: Computational error estimation and adaptive error control for a finite element solution of launch vehicle trajectory problems. SIAM J. Sci. Comput. pp. 1609-1631 (electronic) (1999)

6. Kraft, K., Larsson, S., Lidberg, M.: Using an adaptive FEM to determine the optimal control of a vehicle during a collision avoidance manoeuvre. In: Proceedings of the 48th Scandinavian Conference on Simulation and Modeling (SIMS2007). Linköping University Electronic Press (2007). http://www.ep.liu.se/ecp/027/

7. Meidner, D., Vexler, B.: Adaptive space-time finite element methods for parabolic optimization problems. SIAM J. Control Optim. 46, 116-142 (2007)

8. Pacejka, H.B.: Tyre and Vehicle Dynamics. Butterworth-Heinemann, Oxford (2006) 
APPENDIX

$$
\begin{aligned}
& A=\left[\begin{array}{cccccc}
a_{11} & a_{12} & 0 & 0 & b_{\mathrm{f} 1} & b_{\mathrm{r} 1} \\
a_{21} & a_{22} & 0 & 0 & b_{\mathrm{f} 2} & b_{\mathrm{r} 2} \\
0 & 1 & 0 & 0 & 0 & 0 \\
1 & 0 & V_{X} & 0 & 0 & 0 \\
0 & 0 & 0 & 0 & -\frac{1}{2} & 0 \\
0 & 0 & 0 & 0 & 0 & -1
\end{array}\right], \quad B=\left[\begin{array}{cc}
0 & 0 \\
0 & 0 \\
0 & 0 \\
0 & 0 \\
-\frac{1}{2} & 0 \\
0 & -1
\end{array}\right] \\
& Q_{\text {lane-change }}=\left[\begin{array}{cccccc}
\frac{1}{16} & 0 & 0 & 0 & 0 & 0 \\
0 & \frac{1}{4} & 0 & 0 & 0 & 0 \\
0 & 0 & \frac{1}{0.04} & 0 & 0 & 0 \\
0 & 0 & 0 & \frac{1}{400} & 0 & 0 \\
0 & 0 & 0 & 0 & \frac{1}{4(10 \pi / 180)^{2}} & 0 \\
0 & 0 & 0 & 0 & 0 & \frac{1}{4(\pi / 180)^{2}}
\end{array}\right] \\
& Q_{\text {stabilization }}=6\left[\begin{array}{cccccc}
\frac{1}{16} & 0 & 0 & 0 & 0 & 0 \\
0 & \frac{1}{4} & 0 & 0 & 0 & 0 \\
0 & 0 & \frac{1}{0.04} & 0 & 0 & 0 \\
0 & 0 & 0 & \frac{1}{4} & 0 & 0 \\
0 & 0 & 0 & 0 & \frac{1}{4(10 \pi / 180)^{2}} & 0 \\
0 & 0 & 0 & 0 & 0 & \frac{1}{4(\pi / 180)^{2}}
\end{array}\right] \text {, } \\
& R=\left[\begin{array}{cc}
\frac{1}{4(10 \pi / 180)^{2}} & 0 \\
0 & \frac{1}{4(\pi / 180)^{2}}
\end{array}\right] \text {, }
\end{aligned}
$$

where

$$
\begin{aligned}
a_{11} & =-\left(C_{\mathrm{f}}+C_{\mathrm{r}}\right) / m V_{X}, & & a_{12}=\left(C_{\mathrm{r}} L_{\mathrm{r}}-C_{\mathrm{f}} L_{\mathrm{f}}\right) / m V_{X}, \\
a_{21} & =\left(C_{\mathrm{r}} L_{\mathrm{r}}-C_{\mathrm{f}} L_{\mathrm{f}}\right) / I_{z} V_{X}, & & a_{22}=-\left(C_{\mathrm{f}} L_{\mathrm{f}}^{2}+C_{\mathrm{r}} L_{\mathrm{r}}^{2}\right) / I_{z} V_{X}, \\
b_{\mathrm{f} 1} & =C_{f} / m, & & b f_{\mathrm{f} 2}=C_{\mathrm{f}} L_{\mathrm{f}} / I_{z}, \\
b_{\mathrm{r} 1} & =C_{\mathrm{r}} / m, & & b_{\mathrm{r} 2}=-C_{\mathrm{r}} L_{\mathrm{r}} / I_{z},
\end{aligned}
$$

and with numerical values

$$
\begin{array}{rlrl}
m & =(1500+150) \mathrm{kg}, & & I_{z}=3500 \mathrm{~kg} \mathrm{~m}^{2}, \\
L & =2.755 \mathrm{~m}, & & L_{f}=1.20 \mathrm{~m}, \\
L_{r} & =L-L_{f}, & & \\
C_{f} & =20000 \mathrm{~N} / \mathrm{rad}, & & C_{r}=40000 \mathrm{~N} / \mathrm{rad}, \\
V_{X} & =25 \mathrm{~m} / \mathrm{s}, &
\end{array}
$$

